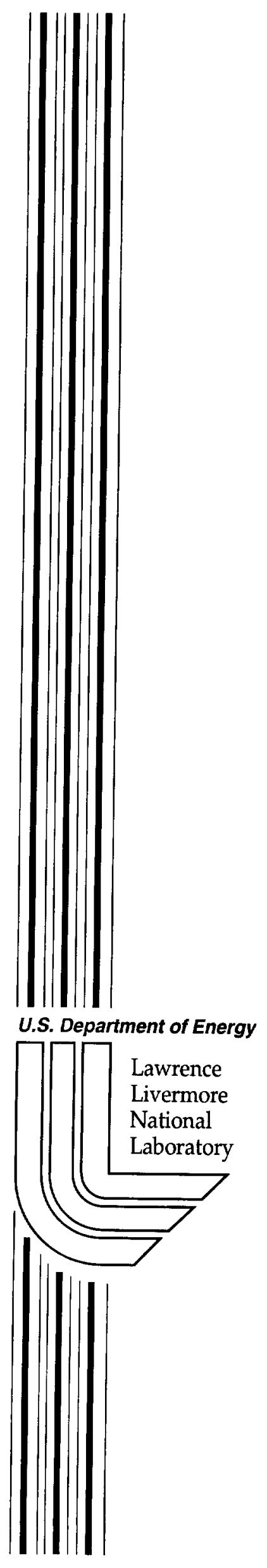

UCRL-ID-147016

\title{
Multiscale Surgical Telerobots
}

R. R. Miles, K. P. Seward, W. J. Benett, F. Tendick, L. Bentley, P. L. Stephan

January 23, 2002 


\section{DISCLAIMER}

This document was prepared as an account of work sponsored by an agency of the United States Government. Neither the United States Government nor the University of California nor any of their employees, makes any warranty, express or implied, or assumes any legal liability or responsibility for the accuracy, completeness, or usefulness of any information, apparatus, product, or process disclosed, or represents that its use would not infringe privately owned rights. Reference herein to any specific commercial product, process, or service by trade name, trademark, manufacturer, or otherwise, does not necessarily constitute or imply its endorsement, recommendation, or favoring by the United States Government or the University of California. The views and opinions of authors expressed herein do not necessarily state or reflect those of the United States Government or the University of California, and shall not be used for advertising or product endorsement purposes.

This work was performed under the auspices of the U.S. Department of Energy by the University of California, Lawrence Livermore National Laboratory under Contract No. W-7405-Eng-48.

This report has been reproduced directly from the best available copy.

Available electronically at http://www.doc.gov/bridge

Available for a processing fee to U.S. Department of Energy

And its contractors in paper from

U.S. Department of Energy

Office of Scientific and Technical Information

P.O. Box 62

Oak Ridge, TN 37831-0062

Telephone: (865) 576-8401

Facsimile: (865) 576-5728

E-mail: reports@adonis.osti.gov

Available for the sale to the public from

U.S. Department of Commerce

National Technical Information Service

5285 Port Royal Road

Springfield, VA 22161

Telephone: (800) 553-6847

Facsimile: (703) 605-6900

E-mail: orders@ntis.fedworld.gov

Online ordering: http://www.ntis.gov/ordering.htm

\section{OR}

Lawrence Livermore National Laboratory

Technical Information Department's Digital Library

http://www.llnl.gov/tid/Library.html 


\title{
Multiscale Surgical Telerobots
}

\author{
R. R. Miles, K. P. Seward, W. J. Benett, F. Tendick, L. Bentley, P.L. Stephan
}

January 23,2002

\section{Abstract}

A project was undertaken to improve robotic surgical tools for telerobotic minimally invasive surgery. The major objectives were to reduce the size of the tools to permit new surgical procedures in confined spaces such as the heart and to improve control of surgical tools by locating positional sensors and actuators at the end effector rather than external to the patient as is currently the state of the technology. A new compact end-effector with wrist-like flexibility was designed. Positional sensors based on MEMS microfabrication techniques were designed.

\section{Introduction}

Minimally invasive surgery (MIS) involves making small incisions in the body, inserting tubes or catheters through the tissue, and placing and manipulating miniature surgical tools inside the body to perform desired tasks. Because it is less traumatic than open surgery where a patient is incised under general anesthesia, MIS provides the benefits of hastened healing and fewer complications with comparable surgical time. The advent of laparoscopic surgery in the 1970 s produced a platform technology that has continually advanced the state of the art of MIS procedures. Minimally invasive surgeons can now perform a host of procedures with only $10 \mathrm{~mm}$ incisions. The further addition of telerobotics to the mix has increased surgical acceptance of MIS by reducing hand tremor and fatigue; allowing more intuitive motions of end effectors; and providing force feedback to the surgeons based on tissue response. Continuation down the path of advancement in surgical telerobotics requires the further shrinking of end effector tools and manipulators. However, a physical size barrier has been reached that makes further downsizing of components impossible without research into microscale and mesoscale sensors and actuators to provide the necessary sensory feedback to surgeons while controlling positions and forces produced by manipulators.

The necessity of further downsizing of surgical devices is not without its tradeoffs. A common problem noted by telerobotics researchers and medical professionals is the ability to transmit the required forces into the body to effectively perform surgeries by minimally invasive means. This is encountered with laparoscopic equipment with diameters smaller than $5 \mathrm{~mm}$ because the shaft leading from the physician's hand to the end effector within the body is compliant and bends under surgical loading conditions. When translated into telerobotics, this poses a problem with the continued shrinking of surgical end effectors in order to enter the body cavity through smaller cannulas or needles.

The further shrinking of end effectors, while keeping surgical efficacy, requires instrumentation designed and engineered to decrease the necessary forces in the body. Ranging from sutures and cutting blades with microscopic tip sharpness to optical and acoustic ablation techniques, new surgical microinstruments are in the research and development phase at several universities and companies and will aid in the abatement of force requirements in future minimally invasive surgery.

In this project, we sought to break the size barrier currently encountered by telerobotics by incorporating micro-electromechanical systems (MEMS) as force and displacement sensors and active material actuators into end effectors with closed-loop control and feedback to surgical 
interfaces. Remote operation of slave robotic surgical instruments using master robots will enable the in situ treatment of medical dysfunctions. Communication bandwidth and robotic autonomy are being developed by means outside of this work. As they are improved, microsurgical robots will be tethered, smart medical instruments that enable true teleoperation across great distances.

Force-feedback telerobotics are necessary not only for surgical acceptance of MIS, but when the communication bandwidth allows, for true tele-surgery. The ability to perform remote emergency care on the battlefield, in rural environments, and in terrestrial or extra-terrestrial exploratory missions is becoming mandatory. To separate highly skilled surgeons from the operation site enables very specified operations to be performed by teams of surgeons hailing from worldwide locales. The imminence of increased computational power will allow these telerobots to be semi- or fully autonomous and leads to the ability for robotic computer-aided surgery in an operating room in rural Kentucky or in one that is on its way to Mars.

\section{Technical Objectives}

Since the late 1970s, minimally invasive surgeries (MIS) have been performed with rigid tools inserted into the body through small incisions (cannulas) and manipulated by surgeons directly. Although MIS greatly reduces patient trauma and decreases hospital stays, surgeons have been slow to adopt MIS techniques for advanced procedures because the constraints of MIS tools greatly reduce dexterity and perception for the surgeon. More recently, surgeons have been able to manipulate these tools through master/slave robotic systems (marketed mainly by Intuitive Surgical, Inc.) While the general trend is moving in the right direction, the full promise of revolutionary telerobotic MIS has not been realized because of the lack of appropriate control systems and scaleable transducers. These constraints make complex operations very difficult or impossible, and therefore require longer periods of time under general anesthesia. Our proposal alleviates these problems through haptic-force feedback and controlled manipulators, providing surgeons with the dexterity and sensation currently missing. This project includes the following fundamental research and development:

miniaturizing surgical end-effectors and manipulator tools to allow a broader range of procedures, 


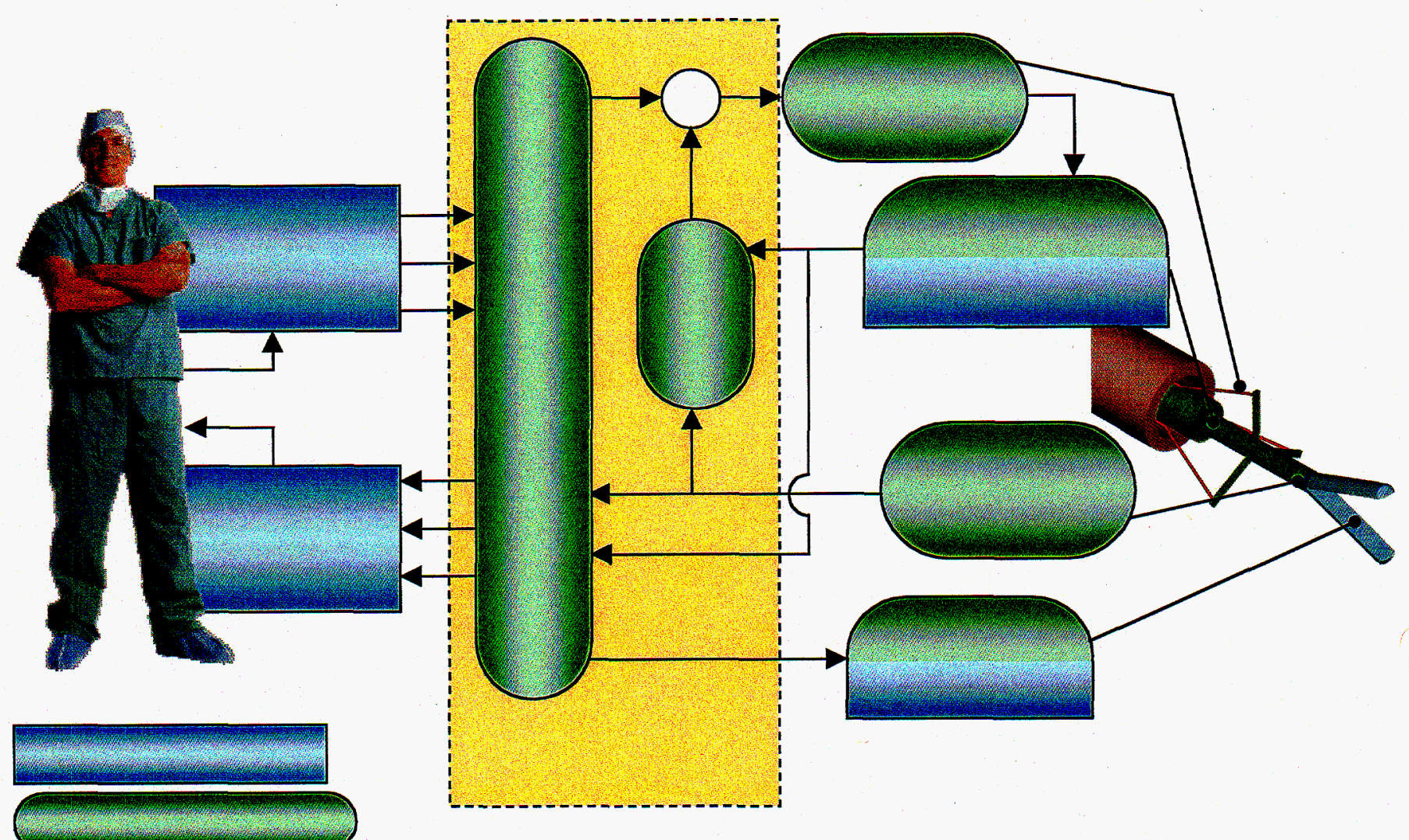

Figure 1: System block diagram

2)

3)

co-locating sensors and actuators on manipulator tools to abate control dynamics,

linking microsystems to the macro-world via integrated mechanical, optical and electrical systems, and

The state of the art of tethered surgical systems and remote operation robotic surgical systems will be expanded to new levels with the funding of this proposal. Development of new MEMS force sensors, mesoscale actuators, and their integration and control lays the research foundation for advanced work leading to surgical demonstrations and integrated micromanipulation systems.

The scope of this project is illustrated by the system block diagram in Figure 1. In this system, the surgeon interacts with the master robot by providing input to sensors and receiving feedback from actuators. Signals are fed through the controller to the slave robot wrist actuator and end-effector. Integrated sensors on the slave manipulator tool feed back the tissue response to the surgeon and control the position of the wrist through the closed-loop controller. We sought to develop the mesoscale wrist-like actuator, and MEMS-based positional sensors. This project was funded for only one out of an anticipated three years. Progress is reflective of this change.

\section{Technical Background}

\section{Limitations of current telesurgical systems}

A major constraint in the design of minimally invasive telesurgical systems is that relatively large force output is often required. We have measured forces necessary to perform common tasks by instrumenting surgical instruments with an ATI 6-axis force torque sensor (unpublished data). These tasks were performed in animal surgeries on adult and infant pigs. The forces we have found range from $500 \mathrm{mN}$ to drive a small needle through the bile duct 
(several $\mathrm{mm}$ in diameter) to several Newtons traction necessary to create sufficient tension to drive a needle in the adult stomach or bladder. In current designs, instead of attempting to insert actuators capable of producing these large forces inside the body power was transmitted to the joints from actuators located outside the patient. In an earlier version of the UCB/UCSF telesurgical workstation, hydraulic actuation was used [1]. Although hydraulics have the advantage of being compact, it is difficult to create backdriveability. Consequently, in the current UCB/UCSF system the wrist DOFs are actuated through tendons by DC motors located outside the patient. Systems developed at SRI International and MIT use similar technology $[2,3]$.

In serial kinematic wrist designs, tendons are wrapped around multiple pulleys. The relatively large forces necessary constrain the minimum diameter of the tendons, and consequently the diameter of the pulleys to minimize friction and fraying of the tendons. The result is a wrist of a minimum total diameter of 8 to $10 \mathrm{~mm}$. This compares poorly to conventional laparoscopic instruments, which range from $5 \mathrm{~mm}$ for instruments designed for the adult abdomen down to $2 \mathrm{~mm}$ for pediatric instruments. Small diameter instruments are necessary to minimize trauma, especially in infants and in thoracoscopic surgery (of the chest) when the instruments must pass between the ribs. Furthermore, smaller size will be necessary to access critical tight spaces. The most significant example is the coronary arteries on the posterior surface of the heart, which are inaccessible with current minimally invasive techniques.

The other major constraint in the design of telesurgical systems is that although the surgeon may be working within a relatively small region, the manipulator must have a large workspace to access that space from outside the patient. Consequently, the manipulator occupies a very large "footprint" next to the operating table as seen in the UCB/UCSF system shown in Figure 2. The problem is magnified by three because manipulators are necessary for the two instruments and another to control the motion of the laparoscope, which must maintain the same orientation relative to the instruments so that the visual-motor relationships perceived by the surgeon are constant. Current designs leave little room near the patient for surgeons, nurses, and equipment. This problem would be magnified in neonatal and pediatric surgery when the telesurgical system would be much larger than the patient, virtually eliminating other access by the operating team.

Fidelity in teleoperation is also limited by the poor force feedback of which current systems are capable. The manipulator, especially the external degrees of freedom, must be stiff for good control, which implies large inertia. Serial kinematic designs require that cables be routed through a circuitous path of pulleys to maintain the constant length of cables passing through proximal joints while destined for distal axes that they control. Each pulley adds substantial friction. Consequently, current systems are at best capable of only 300 to $600 \mathrm{mN}$ of force resolution, which is a significant fraction of the force they are capable of producing. Instead, using miniature parallel kinematic wrists inside the patient would reduce cable routing problems and decrease the inertia of both the micromanipulator and the external manipulator stage, which would be smaller because there would be fewer degrees of freedom externally. 


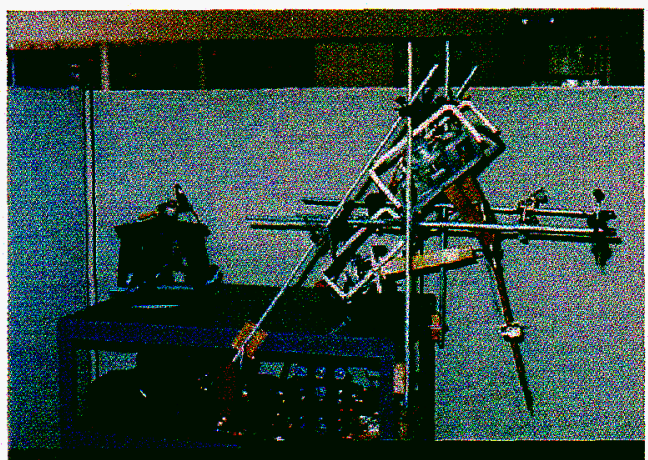

Figure 2. UCB/UCSF Telerobotic system

\section{Technical Progress}

This program was broken down into five areas of technical development. These were the joint design, the positional sensors for the joint design, the actuation design and the control design.

\section{Joint design}

The ideal paradigm for the robotic wrist joint is the human wrist joint. The human wrist is comprised of three rotational degrees of motion co-located at one point. The wrist is able to bend up and down towards the palm, right and left and can be rotated about the axis of the forearm. As such, the human wrist is able to provide a fair degree of mobility in a confined space. This attribute is becoming more important as physicians attempt increasingly difficult procedures on important organs such as the heart. To our knowledge, there is no perfect machine equivalent that satisfies both the requirement for full motion comparable to the wrist and the ability to determine the exact position of the joint. For a example, a simple extension spring can supply the flexibility of the wrist, but each bend also involves combination of rotation and translation which begs the question of where and what kind of sensors would be used to exactly determine the conformation of the spring.

Two joint designs were considered for this research. Neither is perfect but we believe them to be an advance over the existing state-of-the art. The first is a standard universal joint as shown in Figure 3a. This joint has the advantage that it has 2 rotational degrees-of-freedom located at the joint with a third rotation about the forearm. It is capable of 90 degrees flexure but is limited in that it cannot reach all points within a hemisphere centered about the joint. The position of the joint can be accurately determined through use of angular positional sensors placed about the rotational axes.

The second joint design provides the full hemispherical motion of the wrist. The design as shown in Figure $3 \mathrm{~b}$ is comprised of three rods attached to ball-and socket joints which are moved vertically to alter the position of the rod with the fingers attached to them. The advantages to this design are that the full range of wrist motion is available, the actuation mechanism is far easier than with the universal joint, and the vertical motion can be measured with greater precision more easily than the angular position can be measured for the universal joint. The disadvantage with this design is that there will be some flex to the rods leading to some imprecision in location of the fingers. 

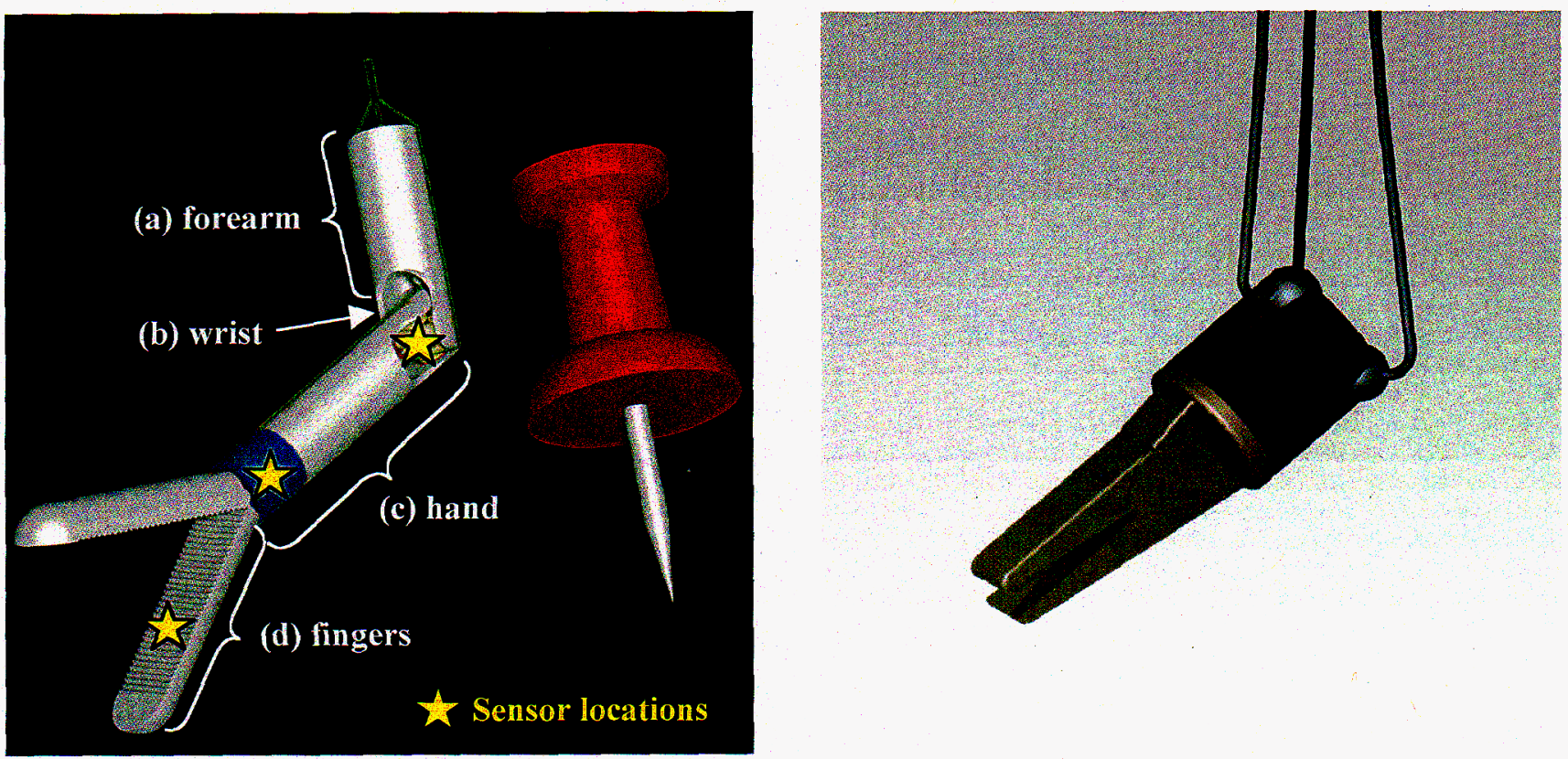

Figure 3: Rendering of al wrist joints (a) based on a universal joint (b) based on three ball \& socket joints.

\section{Positional sensors}

Sensors are necessary in our device to feed back positions and forces acting on end effectors. Due to the miniature components involved in an end effector with a diameter less than $5 \mathrm{~mm}$, all sensors must be manufactured, embedded and integrated by means of microfabrication as seen in Figure 4.

One of the most common rotary sensors is the resistive potentiometer. These are used in
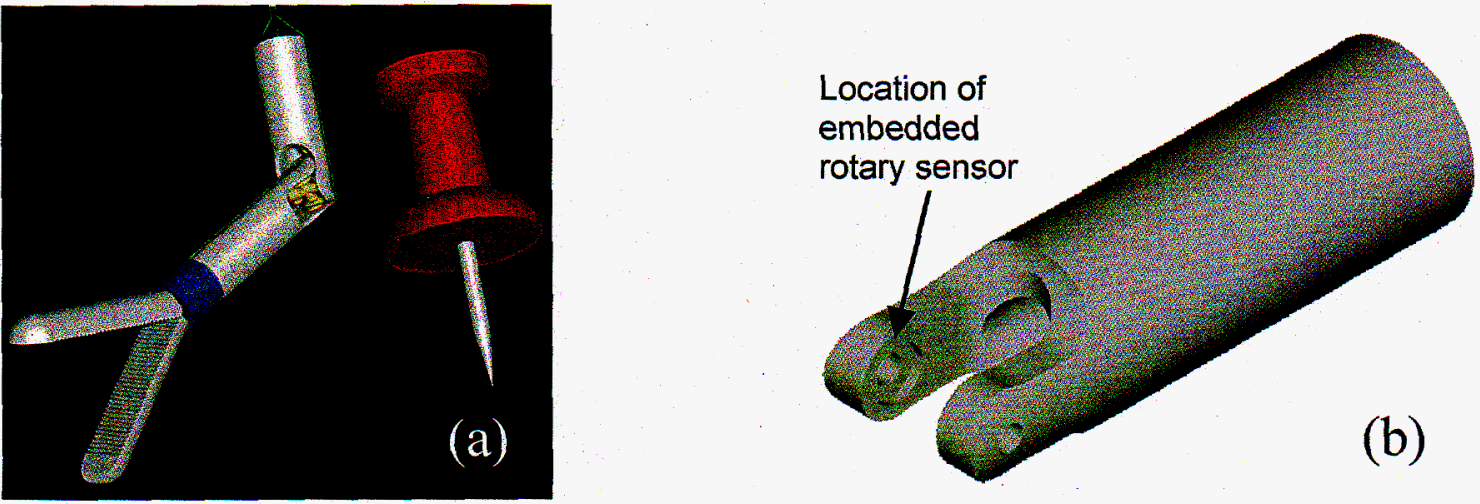

Figure 4: (a) Rendering of a $5 \mathrm{~mm}$ diameter universal joint end effector compared to a common pushpin and (b) one half of the universal joint to show where rotary sensors will be embedded for position sensing.

control knobs on equipment ranging from oscilloscopes to car stereos. A potentiometer is based on the idea of varying a resistance as one contact slides over a resistive link that couples into the rest of the electronics. As current is passed through more of the resistive link, the measured resistance is higher than if current is passed through less of the link. Thus, the movement of the electrode allows for position measurements that can be either linear or angular. While this allows 
for the formation of a simple angular position sensor, the microscale wear, non-linearity and hysteresis encountered with MEMS potentiometers makes their use impractical [4]. Because of these difficulties, coupled resistive-capacitive position sensors have been developed which allow for a full impedance measurement of the constant capacitance and the changing resistance $[4,5]$. To increase the signal from a resistive link, a metal trace resistor is patterned onto a silicon or glass wafer in a serpentine pattern. This increases the resistance change as the sliding contact moves around the circular pattern, but also leads to quantization of the sense resistance. As the contact moves to the next serpentine branch, the resistance changes substantially, but no change in resistance can be resolved unless this larger motion takes place. With current microfabrication techniques, a MEMS resistive-capacitive angular position sensor that is $1.5 \mathrm{~mm}$ across can resolve down to $0.5^{\circ}$, which is our desired resolution. With an end effector that is $20 \mathrm{~mm}$ long, this resolution allows us to detect a change in the end position of $175 \mu \mathrm{m}$. With proper calibration, this allows the $\pm 90^{\circ}$ range in absolute angular position measurement needed for an embedded universal joint sensor. The design for this is shown in Figure 5.

A second sensor was also designed for comparison to the resistive-capacitive sensor. The pure capacitive angle sensor with infinite range [6] is fabricated by patterning two metal crescents onto silicon or glass chips and measuring the differential capacitance between a third, similar metal crescent on the pin in the universal joint and the two on the chip substrate. This differential capacitance measurement is more continuous and linear than a resistive output signal, but relies much more heavily on film thickness uniformity in standoff films that produce the capacitive gap.

The positional sensors for the three rod, ball and socket joint were chosen to be optical interferometric FISO strain gages that would have been embedded in an elastomeric material with one end of the elastomer attached to a reference plane and the other end attached to each rod. The length of linear deflection each rod would thus measured. The position and orientation of the fingers would be a function of the deflections of these rods.
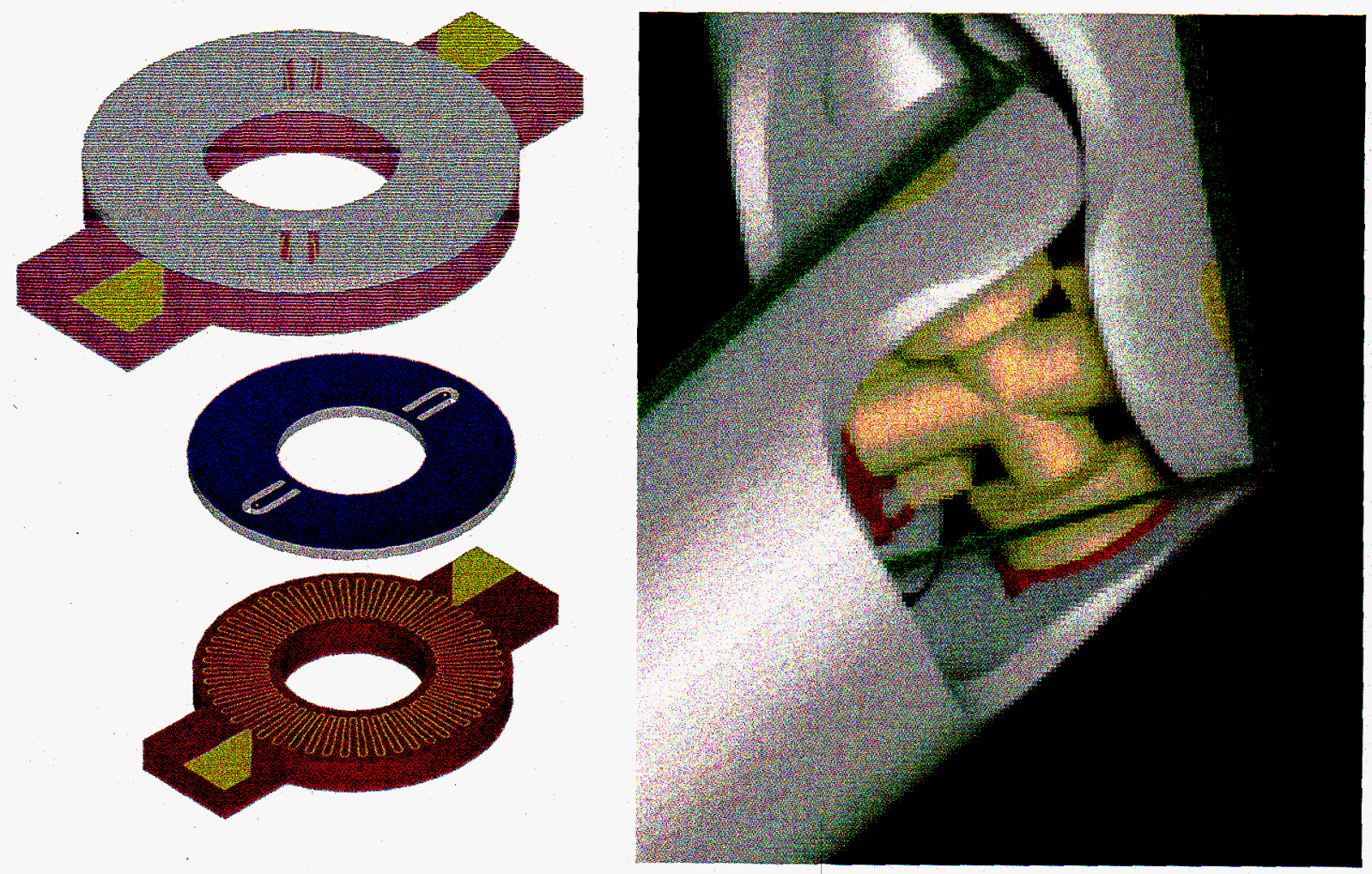

Figure 5: Resistive-capacitive sensor design. 


\section{Active material actuation with shape memory alloys}

Due to the inability to appropriately scale down cable and pulley manipulators, the use of active material actuation in miniature telerobotics is presented as a scalable solution to provide motion and force control. Shape memory alloy fibers are an attractive solution in this system because they provide the highest work output per unit volume of any microactuator alternatives (seen in Figure 6) [7].

Shape memory alloys are appropriately named because they "remember" their shape while undergoing a solid-to-solid phase transformation during heating. At room temperature, certain nickel-titanium alloys (commonly referred to as Nitinol) are in a low-temperature, martensite state. When they are heated beyond a transformation temperature, they undergo the phase transformation to an austenite state. In the martensite phase, the materials exhibit higher

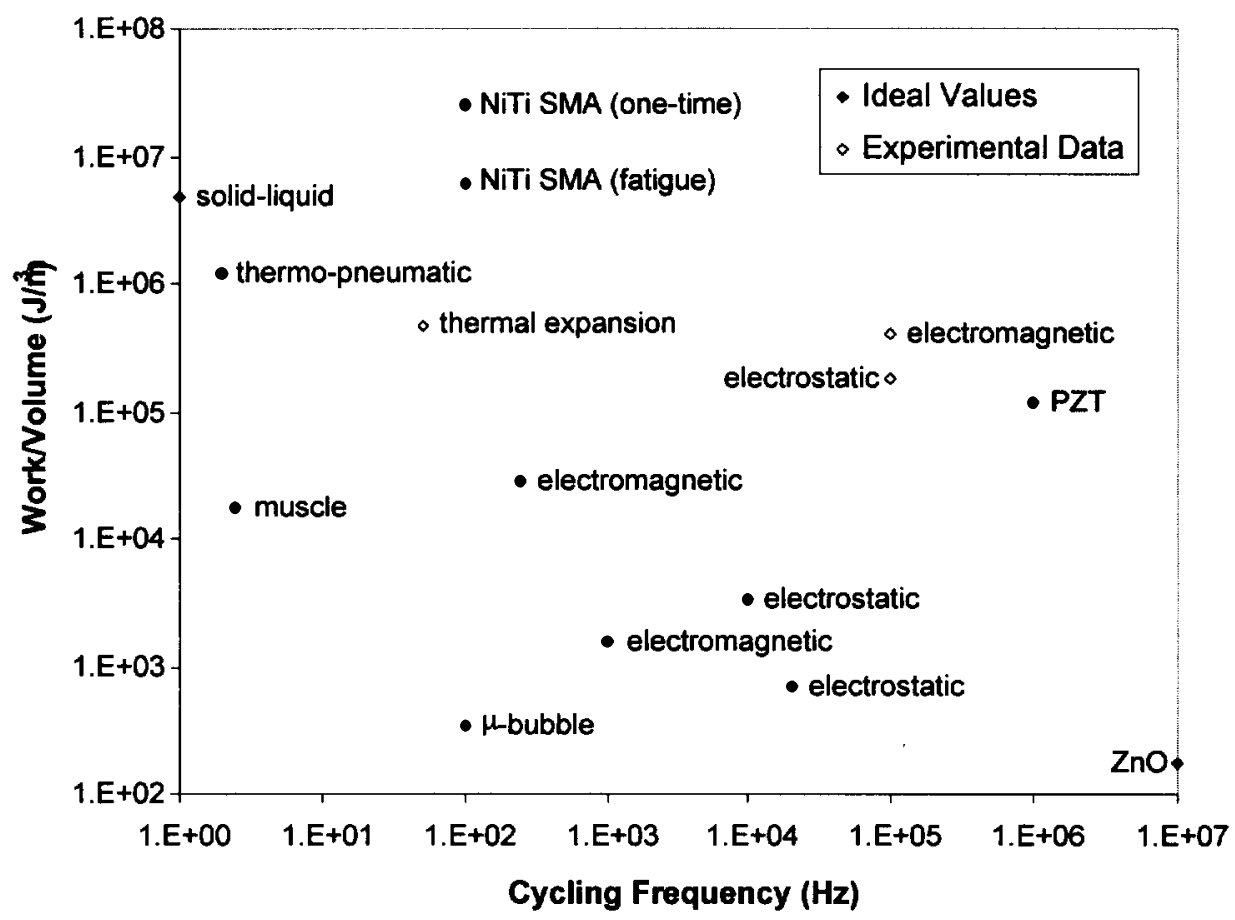

Figure 6: Work output per unit volume vs. operating frequency for several standard microactuators. Note that NiTi SMA provides the highest work density of any actuation method. The listed cycling frequency is given for films rather than fiber material. The cycling frequency of fibers are $\sim 10 \mathrm{~Hz}$ rather than $100 \mathrm{~Hz}$.

ductility than the austenite phase and are therefore easily deformed. When the material is heated it enters the austenite phase (known as reverse transformation). It regains its original shape as long as permanent plastic deformation did not occur in the martensite phase (around $4-10 \%$ strain for most SMAs [8]). Upon cooling to martensite again (martensite transformation), the SMA does not change macroscopic shape unless external loads are applied. In the two-way shape memory effect, both phases remember a unique shape [9-10], which can occur due to residual film stresses changing the shape of the material during the austenite to martensite transformation in the absence of external loading. Electrical resistance changes from the martensite phase to the austenite phase have been observed by other researchers [11-13] and will be exploited in our control systems. 
Phase transformations in the shape memory effect are hysteretic and are therefore characterized by start and finish temperatures for each phase, as illustrated by Figure 7 . In this figure, the four pertinent transformation temperatures are upon heating: the austenite start temperature, $A_{s}$ and the austenite finish temperature, $A_{f}$; and upon cooling: the martensite start temperature, $M_{s}$ and the martensite finish temperature, $M_{f}$. Hysteresis width and transformation temperatures have shown empirical dependence on alloy composition and crystal microstructure, so they are normally consistent as long as fabrication methods do not vary. Results from different researchers are hard to correlate, however, because of different methods of film deposition, annealing conditions, and the effects of different processing steps on film properties.

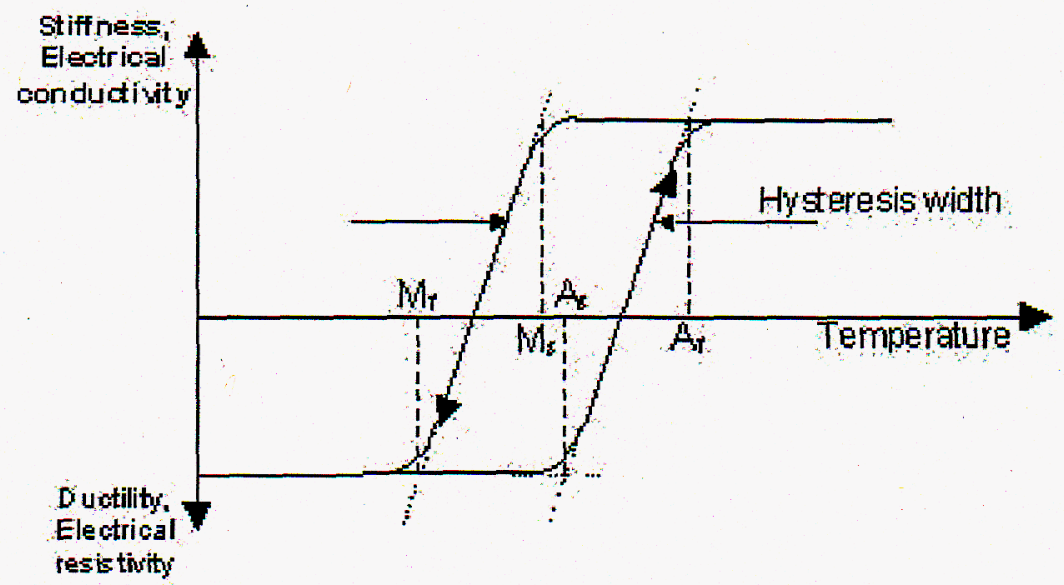

Figure 7: Stiffness and electrical resistivity behavior of NiTi based SMA versus temperature showing a hysteretic behavior between the austenite(high temperature) and martensite (low temperature) phases.

Initial characterization of force and displacement from kinematic arrangements of these fibers was to be tested.

To reduce the scale of actuation for a wrist joint in the telerobot, conventional cable and pulley manipulators must be replaced due to their inherent weaknesses at the mesoscale. At the macroscale DC motors can be used to wind cables around pulleys and create tensile forces that can antagonistically actuate a wrist joint. To mimic this action, SMA fibers can be used without the issues of wrapping, pulley friction, or cable stiffness (all of which lead to complex dynamics that do not allow for control on the mesoscale). When SMA fibers are stretched up to $10 \%$, they will hold the shape with only a small bias against them. When heated by running a current through them, the fibers can recover against a high bias force, creating both force and displacement that are temperature and reaction force dependent. In conventional cable and pulley manipulators, the travel of the cable is only $5-10 \mathrm{~mm}$ to make a wrist move $\pm 90^{\circ}$. Thus, a SMA fiber that runs for only $20 \mathrm{~cm}$ down the shaft of a laparoscopic tube can create the same amount of travel when it is strained only $5 \%$, which is well within the range of repeatable actuation.

An antagonistic actuation system is seen

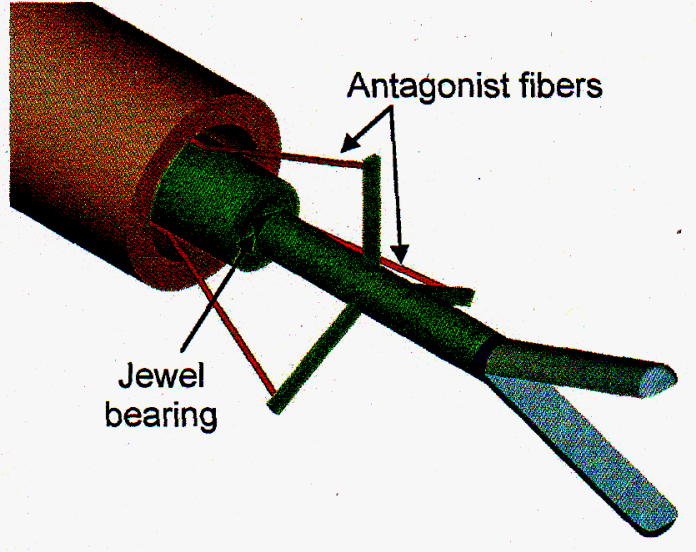

Figure 8: Antagonistic fiber end effector for 2 DOF with jewel bearing kinematics. 
in Figure 8. This system is advantageous because each SMA fiber as stretched applies the necessary bias against the other fibers. When any one Fiber $A$ is heated, it contracts and Fibers $B$ and $\mathrm{C}$ are stretched further, which is possible in their low temperature (off) state. To recover this motion in the other direction, Fiber $\mathrm{A}$ is switched off and Fiber $\mathrm{B}$ and $\mathrm{C}$ are switched on to pull back against the now weak, low temperature bias of Fiber A. When appropriate controls are added to the system, the motion of the wrist will become predictable based on the biases applied by the different actuator fibers. Other orientations have been conceived that will be tested to use four antagonistic fibers to create 3 DOF: 2 rotations about the intersecting axes of the wrist joint plus a slight roll, or pronation, of the wrist.

In order to control SMA fiber actuation, we must characterize the necessary mechanical behavior of the fibers using an Instron mini-tensile load frame with an attached environmental chamber for ambient heating and cooling of the fiber. With the abilities to apply ambient heat with the environmental chamber or Joule heating with a current through the fiber, we would be able to discriminate between thermal effects and electrical effects in the alloy. Characterization would be made of stress versus strain for several ambient temperatures, which would be correlated to stress versus strain for several applied currents to translate the applied currents into actual heating of the fibers. This would be compared with Joule's Law and Newton's Law of Cooling in the cases of forced and free convective cooling of the fibers. Shape memory alloys also obey a Clausius-Clapeyron relationship, which changes transformation temperatures based on applied loads. We would therefore measure the resistance of the fibers during these tests to view the resistivity change that takes place during the alloys' solid-to-solid phase transformation. This resistivity change would then be incorporated into control models of the antagonistic actuator to serve as an indication of phase change.

Actuation schemes under consideration for the SMA fibers include two-stage relay controllers, sliding mode controllers, and series and parallel recruitment controllers. The twostage relay controllers operate as simple, robust on/off controllers that can actuate one fiber at a time to produce motion and force. A sliding mode controller would allow stable current control of each fiber while temperature limitations would protect against breaking or overheating the fibers. This configuration would also allow fibers to actuate against other fibers, producing stable forces in the case when a load needs to be held in place. Series controllers allow different sections along the length of a stretched fiber to be heated separately. In this case, if only $10 \%$ of the total displacement possible from a fiber is desired, only $10 \%$ of the length of the fiber would be heated. Finally, parallel recruitment control schemes involve the routing of parallel fibers and the actuation by current of only one fiber in the bundle. As more stress is placed on the actuated fiber, its resistance will increase, causing more heat to be produced by a fixed current. This heat will affect neighboring fibers, "recruiting them," and additional force will be created by the fiber bundle to counteract imposed stresses. This is the most biomimetic control scheme, acting in a similar manner to human muscle tissue.

A testbed for evaluation of SMA control algorithms, was design and fabricated as shown in Figure 9. The testbed is a 1 DOF rotary joint actuated by an antagonistic pair of SMA fiber actuators. Position and force feedback are provided by an angular displacement sensor and two fiber tensile force sensors. Forced air cooling will be used. A commercially available haptic input device, the PHANToM, would be constrained to one degree of freedom and used as the master manipulator.

The complete controller will include the outer teleoperation control loop and a slave side local linearizing control loop. The nonlinearities of the actuator would be compensated for in the local control loop, and elements of linear control theory applied in analysis of the teleoperation loop. Other researchers have used methods from nonlinear control theory to achieve good position and force tracking at low bandwidths with various SMA actuators.(Dickinson and Wen 1998; Grant and Hayward 2000) These have included feedback linearization and a robust relay 
controller implemented with an antagonistic actuator configuration. We would have used a modified version of this relay controller and actuator model as the starting point for the linearizing controller.

The teleoperator design methodology is based on a two-port network modeling approach as shown in Figure 10. This approach treats the master and slave effort and flow variables as the inputs and outputs of the system, and analyzes the matrix relating these variables. Important parameters of the system, including free space tracking and transmitted impedances, can be evaluated using this approach. Applying two-port modeling to the possible teleoperation architectures, and using models of the sensor and actuator characteristics, we will compare the architectures. After determining the optimal architectures, the controller implementation will be evaluated and modified based on stability and robustness considerations. We have implemented simulations in Matlab, incorporating known SMA parameter values and the Grant algorithm, to tune the controller parameters. The simulation will also be used to evaluate the model, which is crucial to understanding the experimental results.

We have submitted two relevant papers for publication, one of which has already been accepted. The first details our teleoperator design methodology [14]. The second uses previously recorded data of expert surgeons suturing and tying knots. This data is fed into the inverse kinematics of two fundamental wrist designs (roll-pitch-roll and roll-pitch-yaw) to determine which design is best for the kinematic constraints of different surgical procedures [15].

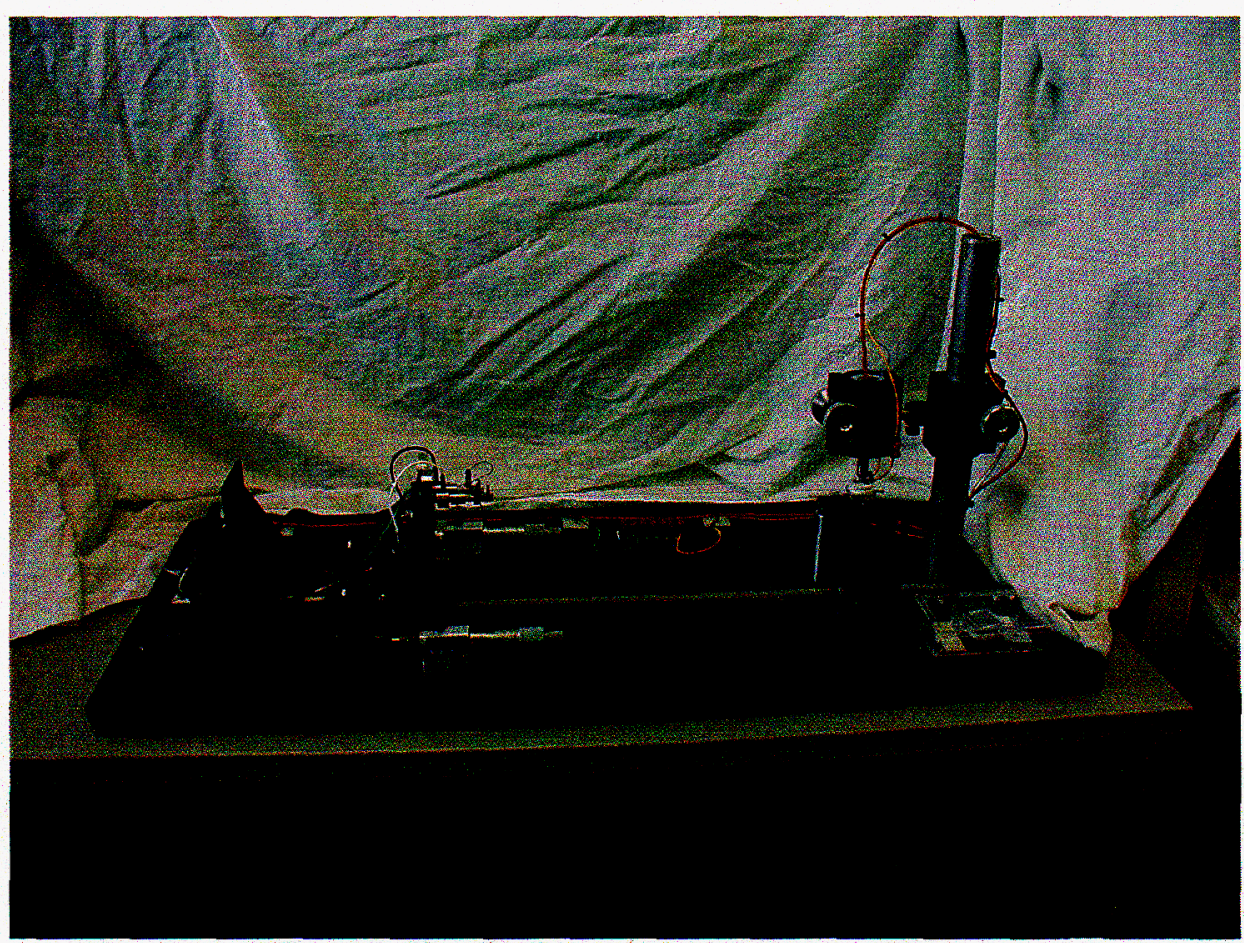

Figure 9a. SMA actuator testbed. 


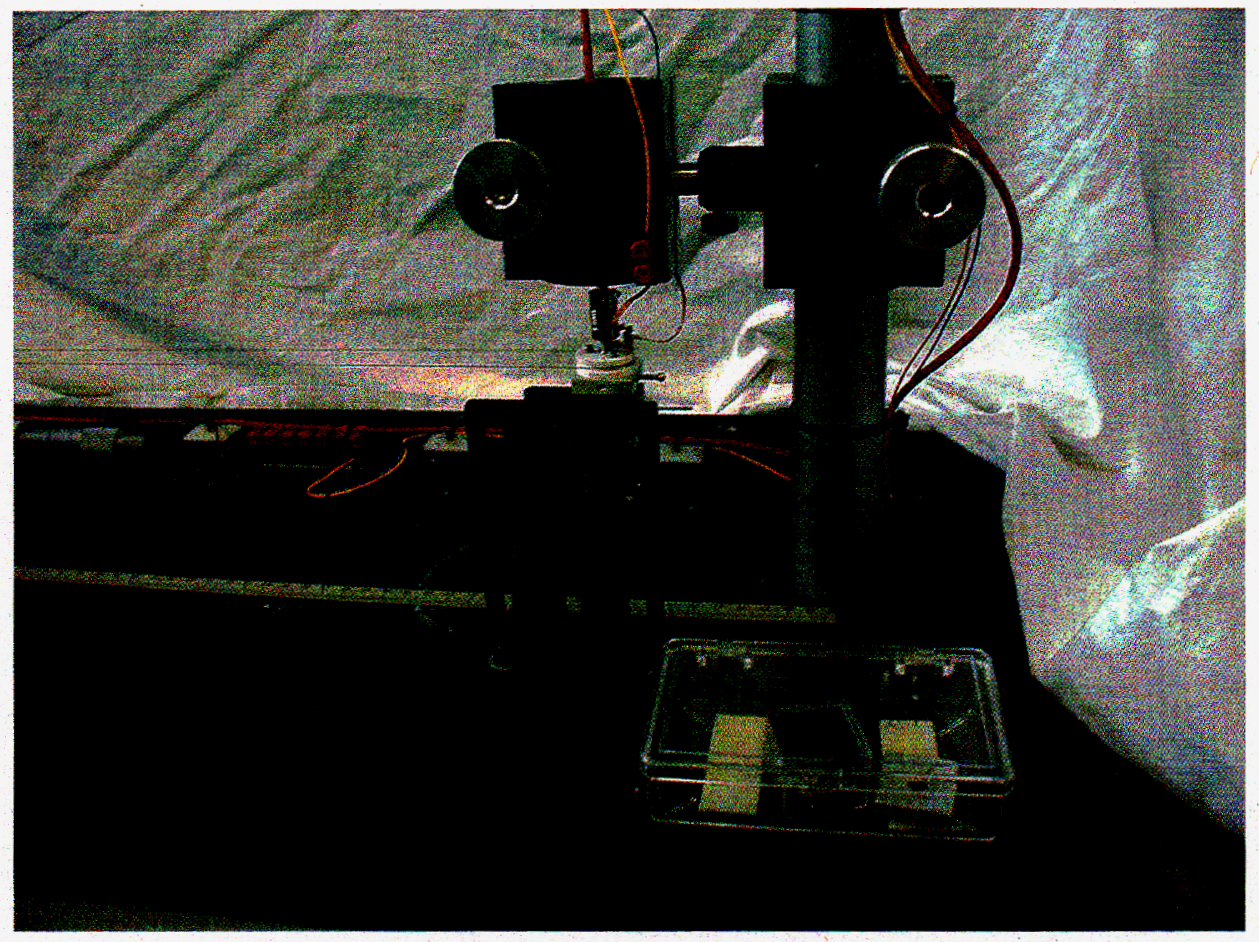

Figure 9b. Close-up of testbed, showing SMA fiber pair wrapped around ceramic disk, with capacitive angular sensor.

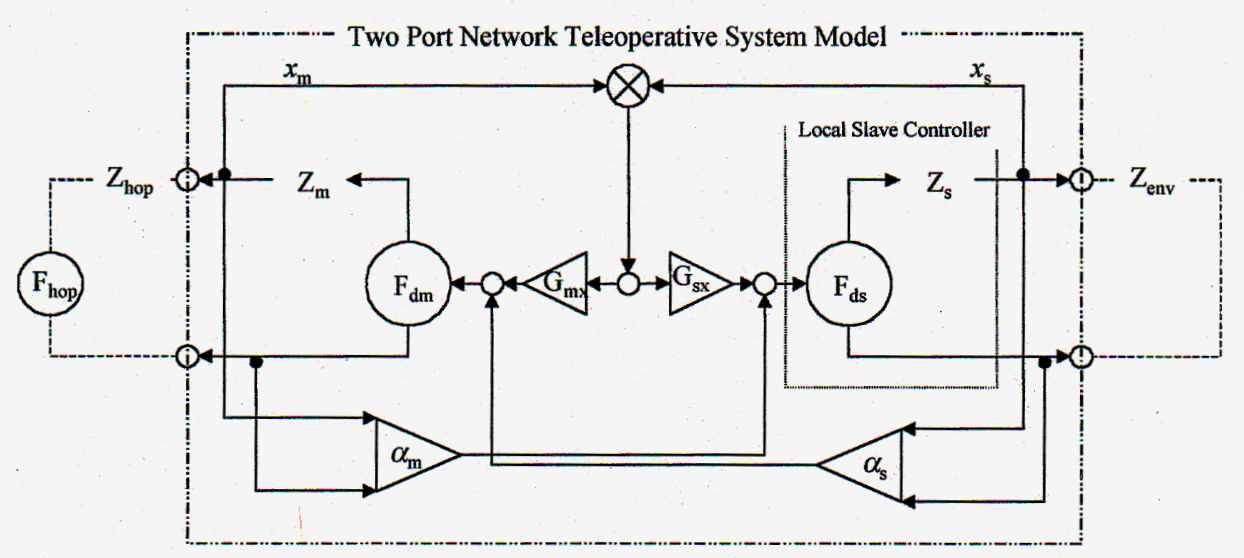

Figure 10. Two-port teleoperator controller architecture.

\section{Summary}

Th first year of a three year telerobotics project was completed with advances made in the areas important to building the next generation surgical robotic systems. Specific achievements include - a novel design for a compact, highly flexible joint design for surgical robotic end effectors capable of emulating human wrist motion, 
- design of micro sensors to be embedded at the end effector thus dramatically increasing the controlability of surgiacl end -effectors over what is currently available and

- exploration into use of local actuation methods to reduce the size and increase control over surgical robotic manipulation.

\section{Literature Cited}

1. M. C. Cavusoglu, F. Tendick, M. Cohn and S. S. Sastry, "Control of a Telesurgical Workstation," IEEE Trans. on Robotics and Automation, v15, no4, pp. 728-739, Aug 1999.

2. J. W. Hill, P. S. Green, J. F. Jensen, Y. Gorfu and A. S. Shah, "Telepresence surgery demonstration system," Proc. IEEE Intl. Conf. Robotics and Automation, San Diego, pp. 2302-7, 1994.

3. A.J. Madhani, "Design of teleoperated surgical instruments for minimally invasive surgery," Ph.D. Thesis, Massachusetts Institute of Technology, 1998.

4. X. Li and G.C.M. Meijer, "A Novel Smart Resistive-Capacitive Position Sensor," IEEE Transactions on Instrumentation and Measurement, v44, no3, pp. 768-770, June 1995.

5. X. Li and G.C.M. Meijer, "A Novel Low-Cost Noncontact Resistive Potentiometric Sensor for the Measurement of Low Speeds," IEEE Transactions on Instrumentation and Measurement, v47, no3, pp. 776-781, June 1998.

6. R.D. Peters, “Capacitive angle sensor with infinite range," Rev. Sci. Instrum., v64, no3, pp. 810-813, March 1993.

7. P. Krulevitch, A.P. Lee, P.B. Ramsey, J.C. Trevino, J. Hamilton and M.A. Northrup, "Thin Film Shape Memory Alloy Microactuators," Journal of Microelectromechanical Systems, Vol. 5, No. 4, pp. 270-282, December 1996.

8. J.B. Kennedy, (translator), Shape Memory Alloys, H. Funakubo, Ed., New York: Gordon and Breach, 1984.

9. J. Perkins and D. Hodgson, "The Two-Way Shape Memory Effect," Engineering Aspects of Shape Memory Alloys, T. W. Duerig, K. N. Melton, D. Stockel and C. M. Wayman, Eds., London, U.K.: Butterworth-Heinemann, pp. 195-206, 1990.

10. M. Sato, A. Ishida and S. Miyazaki, "Two-Way Shape Memory Effect of Sputter-Deposited Thin Films of Ti 51.3 at.\% Ni," Thin Solid Films, Vol. 315, pp. 305-309, 1998.

11. A. D. Johnson, "Vacuum-deposited TiNi shape memory film: characterization and applications in microdevices," Journal of Micromechanics and Microengineering, Vol. 1, pp. 34-41, 1991.

12. H. C. Ling and R. Kaplow, "Stress-Induced Shape Changes and Shape Memory in the R and Martensite Transformations in Equiatomic NiTi," Metallurgical Transactions A, Vol. 12A, pp. 2101-2111, December 1981.

13. H. C. Ling and R. Kaplow, "Phase Transitions and Shape Memory in NiTi," Metallurgical Transactions A, Vol. 11A, pp. 77-83, January 1980.

14. M.C. Cavusoglu, A. Sherman, and F. Tendick, "Design and Experimental Evaluation of Bilateral Teleoperation Controllers for Telemanipulation in Soft Environments." Submitted to IEEE Transactions on Robotics and Automation, June 2001.

15. M.C. Cavusoglu, I. Villanueva, and F. Tendick, "Workspace Analysis of Robotic Manipulators for a Teleoperated Suturing Task." IEEE/RSJ International Conference on Intelligent Robots and Systems (IROS 2001). In press. 